\title{
INDUCED OPTICAL ACTIVITY IN RHODOPSIN ANALOGS
}

Edward M. Johnston and Robert Zand

\begin{abstract}
Biophysics Research Division, Institute of Science and Technology, and Department of Biological Chemistry, The University of Michigan, Ann Arbor, Michigan 48105
\end{abstract}

Received April 10, 1972

SUMMARY

The Schiff's base derived from 11-cis retinaldehyde and (S)- - -(1-naphthyl)ethylamine has been synthesized and its $\overline{C D}$ spectrum measured. Schiff's bases derived from all-trans retinaldehyde and (S) $-\alpha-(1-n a p h t h y l)$ ethylamine, (S) $-\alpha-$ phenylethylamine and (R) - 2,2'-diamino-6,6'-dimethylbiphenyl were also prepared and their $C D$ spectra recorded. The introduction of optical activity into the retinaldehyde moiety of these simple Schiff's bases could be explained in terms of a coupled oscillator mechanism. These results require that previous explanations of the $500 \mathrm{~nm}$ Cotton effect of rhodopsin be re-examined.

The visual pigment rhodopsin from different animal species exhibits circular dichroic absorption bands in the wavelength regions of 500 and $350 \mathrm{~nm}$. 1-6 The $500 \mathrm{~nm}$ extrinsic cotton effect can be assigned to the electronic transition that occurs in the conjugated chromophore at this wavelength, and is sometimes referred to as the $\alpha$ band. Since 11 -cis retinaldehyde is optically inactive, a mechanism for the induction of the extrinsic cotton effect in rhodopsin is required.

Crescitelli, Mommaerts and Shaw $^{1}$ have proposed that the optical activity is a consequence of a spatial distortion imposed on the chromophore by the protein moiety of the molecule. Other investigators ${ }^{2,7}$ have generally followed this idea, suggesting in one case ${ }^{7}$ that the chromophore was most likely twisted at the $\mathrm{C}_{11}-\mathrm{C}_{12}$ double bond. Mommaerts ${ }^{8}$ has argued that 11-cis retinaldehyde can assume one of two possible enantiomeric forms and that the observed circular dichroism of rhodopsin arises through the preferential stabilization of one of these forms by the protein. Such an argument for the origins of the observed circular dichroism in rhodopsin cannot be used to explain the optical activity 
in isorhodopsin ${ }^{2}$ which contains the 9-cis retinaldehyde isomer. Moreover, the hypothesis does not work in the case of the metarhodopsins which contain the all-trans isomer as the chromophore responsible for absorption in the visible region. ${ }^{6}$ The spatially distorted chromophore hypothesis was also utilized by Kito, Azuma and Maeda ${ }^{3}$ to account for their circular dichroism data on squid rhodopsin.

We report the results of our experimental and theoretical studies on the induction of optical activity into all-trans and ll-cis retinaldehydes via Schiff's base formation with simple aromatic, optically active amines. The structure of one of these Schiff's bases is shown in Figure 1 . Whatever the mechanism for the introduction of optical activity into these simple analogs of rhodopsin, it cannot be based on a twisted chromophore hypothesis since the amine component of the Schiff's base is too small to impose any spatial distortion upon the retinaldehyde.

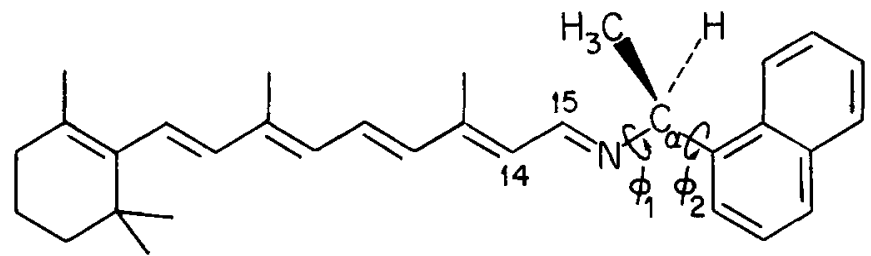

Figure 1. Illustration of the Schiff's base (S)-N-all-trans retinylidene $\alpha-$ (1-naphthyl) ethylamine showing the rotational angles $\phi_{1}$ and $\phi_{2}$.

A view capable of explaining both the rhodopsin data and our data is that the chromophore becomes optically active through a kirkwood-type coupled oscillator mechanism. In this approach, the transitions of the retinaldehyde chromophore are considered to interact with the transitions of the nearby aromatic side chains and as a consequence of this interaction optical activity is induced into the retinaldehyde chromophore. ${ }^{6}$

A similar coupled oscillator approach has been successfully applied to the calculations of the rotational strength of the Soret transition of the heme moiety in myoglobin. 10,11 
We have synthesized Schiff's bases by reacting all-trans retinaldehyde with the following optically active amines: (I) (S)- - -(1-naphthyl)ethylamine, (2) (S)- $\alpha$-phenylethylamine and (3) (R) $-2,2^{\prime}$-diamino-6,6'-dimethylbiphenyl.

\section{MATERIALS AND METHODS}

The optically active amines $(\underline{S})-\alpha-(1-$ naphthyl) ethylamine and $(\underline{S})-\alpha-$ phenylethylamine were purchased from the Norse Laboratories, Inc., Santa Barbara, California. All-trans retinal was purchased from Eastman organic Chemicals, Rochester, New York. The 11-cis retinal was generously provided by Paul Brown of Harvard University and also by Hoffman-LaRoche Co. The (+) $2,2^{\prime}-$ diamino-6, $6^{\prime}$ dimethylbiphenyl was synthesized and resolved by the method of Meisenheimer and Horing. 12

A typical preparation of a Schiff's base was to react about $15 \mathrm{mg}$ of the retinaldehyde with a $30 \%$ excess of the amine in $1 \mathrm{ml}$ of dry chloroform. The mixture was allowed to stand overnight at room temperature in the dark. The solutions were then diluted about 1000 to 1 with isooctane for spectral measuraments. Completion of the reaction was verified by diluting an aliquot of the reaction mixture with chloroform and then treating with HCl gas. The presence of unreacted retinaldehyde is indicated by a peak at $390 \mathrm{~nm}$.

Absorption spectra were recorded on a Cary 15 spectrophotometer. CD spectra were recorded on a JASCO ORD/CD SS-20 spectropolarimeter. Calculations of conformational energy and rotational strengths were performed on a Digital Equipment Corp. PDP-10 computer.

\section{RESULTS AND DISCUSSION}

The three Schiff's bases prepared from all-trans retinaldehyde and amines 1, 2 and 3 displayed Cotton effects in the region of retinaldehyde absorption. For this report, however, we confine our discussion to the amine, $(\underline{S})-\alpha-(1-$ naphthy 1) ethylamine and its Schiff's base (Figure 1). The amine exhibits two main optically active transitions, $a{ }^{l_{L_{a}}}$ band at $282 \mathrm{~nm}$ and $a^{{ }^{1}}{ }_{B_{b}}$ band at $224 \mathrm{~nm}$. The Schiff's base with all-trans retinaldehyde has an additional op- 


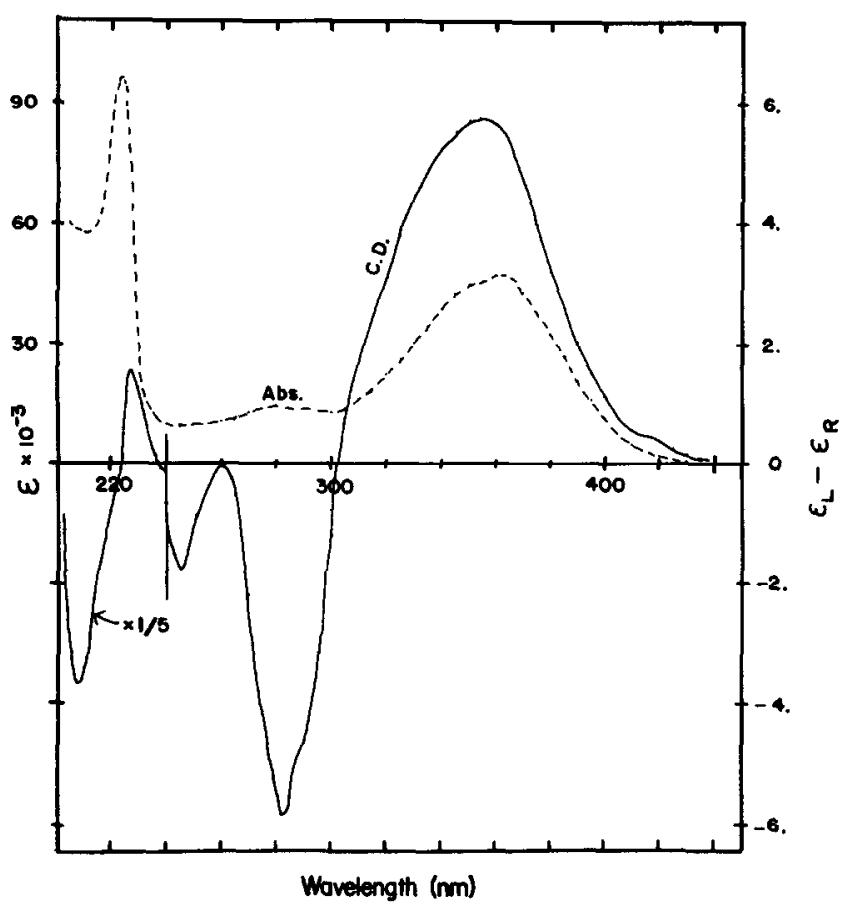

Figure 2. Absorption spectrum ----- and circular dichroism of the (S) $\mathrm{N}$-all-trans retinylidene $\alpha$-(1-naphthyl) ethylamine in isooctane solution. The solution contains a $30 \%$ excess of free amine.

tically active band at $360 \mathrm{~nm}$ in isooctane solution as shown in Figure 2. The band has a rotational strength of +0.27 DBM (Debye Bohr Magnetons). This is of the same magnitude as the rotational strength of +0.52 DBM that has been reported $^{6}$ for the $500 \mathrm{~nm}$ band of cattle rhodopsin. This Schiff's base also shows induced optical activity in the region of naphthyl absorption. The Cotton effects in this wavelength region occur at $282 \mathrm{~nm}, 246 \mathrm{~nm}, 227 \mathrm{~nm}$ and $208 \mathrm{~nm}$. The compound can be protonated by passing dry gaseous $\mathrm{HCl}$ through a chloroform solution of the Schiff's base. When this is done the absorption and circular dichroic peaks near $360 \mathrm{~nm}$ are shifted about $100 \mathrm{~nm}$ towards the red (Figure 3 ).

In an attempt to account for the induced optical activity of this simple visual pigment analog, the rotational strengths of the molecule were calculated using coupled oscillator theory. In carrying through this calculation a con- 


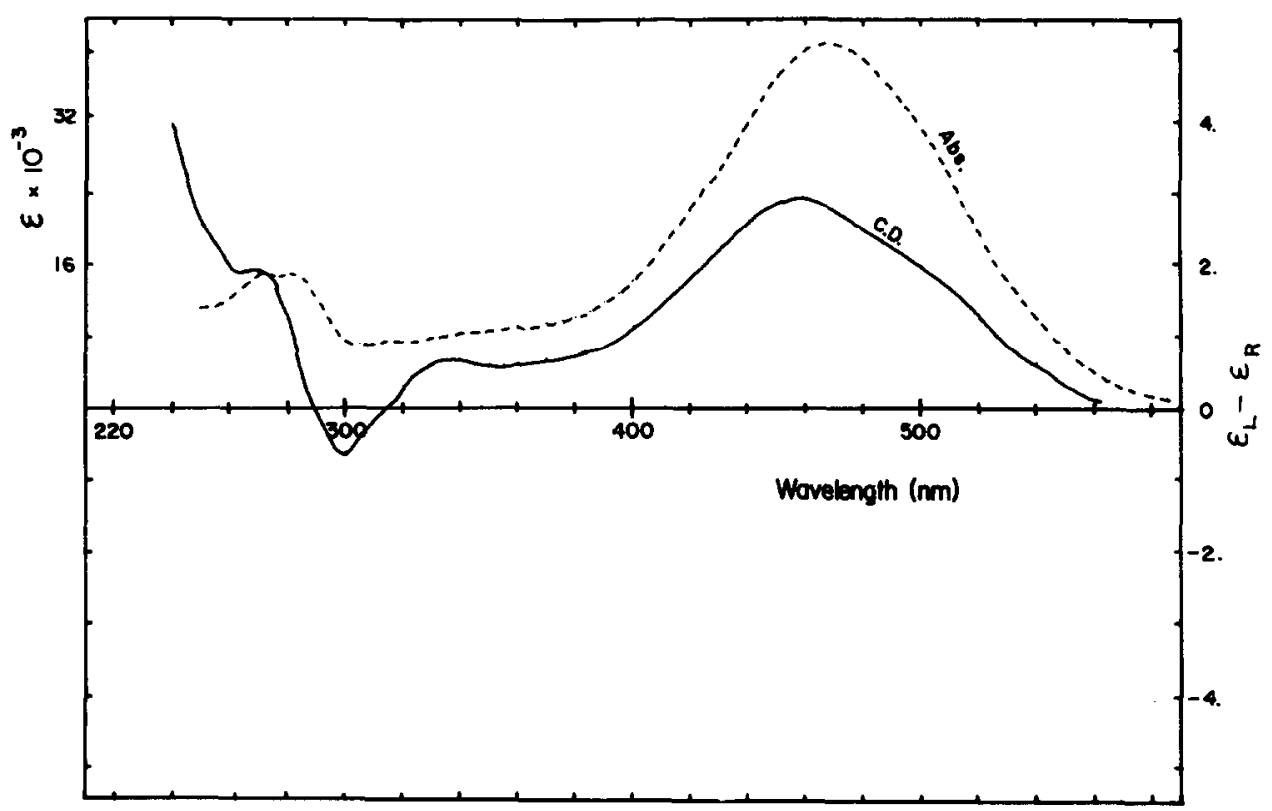

Figure 3. Absorption spectrum ----- and circular dichroism of the protonated form of the Schiff's base (S)-N-all-trans retinylidene $\alpha-$ (1-naphthyl) ethylamine in chloroform solution, with a $30 \%$ excess of amine.

formational energy map was initially prepared to determine the range of allowed values for the two rotation angles $\phi_{1}$ and $\phi_{2}$ (Figure 1). The symbol $\phi_{1}$ denotes the $\mathrm{N}-\mathrm{C}_{\alpha}$ rotation and $\phi_{2}$ denotes the $\mathrm{C}_{\alpha}-\mathrm{C}_{\text {naphthy }}$ rotation. Each angle is taken to be zero in the s-cis conformation. The second step in the calculation was to derive free electron molecular orbital (FEMO) wave functions for the ground state and the relevant excited states of the retinylidene and naphthyl groups. Utilizing the monopole method, ${ }^{13}$ and the FEMO wave functions, a value for the Coulomb matrix element between two transitions a and b was obtained This parameter along with other parameters could now be substituted into equation 1. This equation for the induced rotational strengths that result from the coupling of electrically allowed, magnetically forbidden transitions was derived by Tinoco. ${ }^{14}$ The form given below is appropriate for the case of rhodopsin and its analogs.

$$
R_{i a}=\frac{2 \pi}{c} \quad \sum_{j \neq i} \sum_{b \neq a} \frac{V_{i 0 x i j 0 b} \nu_{a} \nu_{b} \overrightarrow{\mathrm{R}}_{i, j} \cdot\left(\vec{\mu}_{j o b} \times \vec{\mu}_{i o a}\right)}{h\left(\nu_{b}^{2}-\nu_{a}^{2}\right)}
$$


where $R_{i a}$ is the rotational strength induced in transition $a$ of the $i$ th group, $V_{i o a ; j o b}$ is the Coulomb matrix element between the two transitions, $\overrightarrow{\mathrm{R}}_{i j}$ is the vector joining the centers of the groups, and $\vec{u}_{j o b}$ is the electric transition dipole moment of transition $b$ of the $j$ th group.

When the two rotation angles $\phi_{1}$ and $\phi_{2}$ are set at $180^{\circ}$ and $300^{\circ}$ respective: $l_{Y}$, all four of the calculated rotational strengths agree within a factor of two with the experimentally determined rotational strengths. The calculated spectrum did not exhibit the Cotton effects at $246 \mathrm{~nm}$ and $208 \mathrm{~nm}$ because no assignable Lransitions could be found in the absorption spectrum at these wavelengths. The rotational strength that was predicted to occur at $167 \mathrm{~nm}$ could not be measured experimentally. It was estimated from the measured rotational strengths by means of the Kuhn sum rule. ${ }^{15}$ A comparison of calculated and experimental data is presented in Table 1.

The results of this investigation indicate that the $490 \mathrm{~nm}$ Cotton effcct of rhodopsin is not prima facie evidence for the operation of the distorted chromophore hypothesis. Our data indicate that the retinaldehyde transitions may become optically active by coupling with the transition of aromatic side chains located within a $15 \AA$ radius of the chromophore.

The Schiff's base of $11-\underline{\text { cis }}$ retinal and $(\underline{s})-\alpha-(1-$ naphthyl)ethylamine was also prepared and studied. This compound exhibits an extrinsic cotton effect in the region of $360 \mathrm{~nm}$ which is less intense and displays a different shape than that of the all-trans isomer. This behavior suggests that there may be conformational mobility about the $\mathrm{C}_{12}-\mathrm{C}_{13}$ single bond in this retinaldehyde isomer.

Schiff's bases formed from L-Iysine or L-phosphatidylethanolamine were optically inactive in the visible wavelength region. The Schiff's base of all-trans retinaldehyde and poly-L-1ysine displays extrinsic cotton effects and these spectra are currently being analyzed.

The results of this investigation suggest that optical activity in the retinaldehyde chromophore of rhodopsin and these rhodopsin analogs may be 


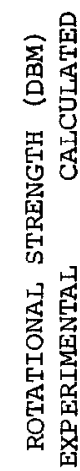

0
$\stackrel{0}{+}$
$\stackrel{0}{+}$
+

$\begin{array}{ll}0 & m \\ 0 & 0 \\ 1 & -1 \\ 0 & 0\end{array}$

\begin{tabular}{l|l}
-1 & 1 \\
0 & 1 \\
0 & 1
\end{tabular}

\begin{tabular}{ll}
0 & \multirow{1}{1}{} \\
$\because$ & 0 \\
1 & 0
\end{tabular}

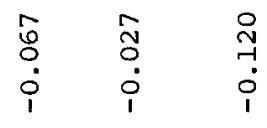

$\infty$
0
$\stackrel{0}{0}$
$\dot{0}$
+

ญै

$$
\text { i্c }
$$

皇息

$\stackrel{\circ}{0}$

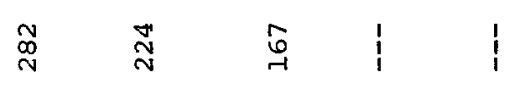

峁

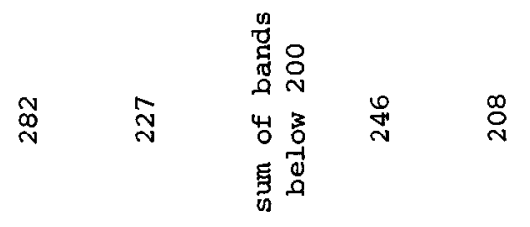

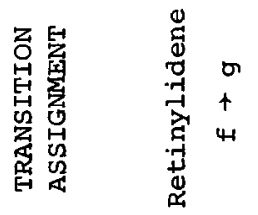

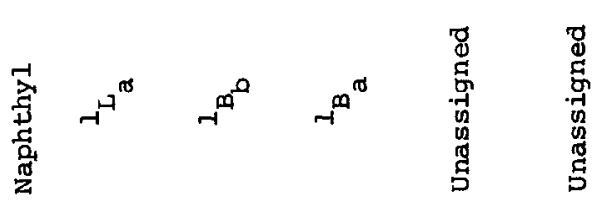


best accounted for by a coupled oscillator mechanism rather than the twisted chromophore hypothesis.

This work was supported by the National Institutes of Health through grants GM-1355, GM-14035 and NB-05036.

\section{REFERENCES}

1. Crescitelli, F., Mommaerts, W. F. H. M., and Shaw, T. I., Proc. Nat. Acad. Sci. U. S. 56, 1729 (1966).

2. Takezaki, M., and Kito, Y., Nature 215, 1197 (1967).

3. Kito, Y., Azuma, M., and Maeda, Y., Biochim. Biophys. Acta 154,352 (1968).

4. Schichi, H., Lewis, M. S., Irreverre, F., and Stone, A., J. Biol. Chem. 244, 529 (1969).

5. Horwitz, J., and Heller, J., Biochemistry 10, 1402 (1971).

6. Waggoner, A. S., and Stryer, L., Biochemistry 10, 3250 (1971).

7. Daemen, F. J. M., and Bonting, S. L., Nature 222, 879 (1969).

8. Mommaerts, W. F. H. M., in The Retina: Morphology, Function and Clinical Characteristics (Straatsma, B. R., Hall, M. O., Allen, R. A., and Crescitelli, F.), 225, University of California Press, LosAngeles (1969).

9. Kirkwood, J. G., J. Chem. Phys. 5, 479 (1937).

10. Hsu, M.-C., and Woody, R. W., J. Am. Chem. Soc. 91, 3679 (1969).

11. Hsu, M. C. , and Woody, R. W., J. Am. Chem. SOC. $\overline{\text { A3 }}, 3515$ (1971).

12. Meisenheimer, J., and Horing, $\bar{M}, \bar{B}$ er. $60 \mathrm{~B}, \overline{1425}$ (1927).

13. Haugh, E. F., and Hirschfelder, J. O., J. Chem. Phys. 23, 1778 (1955).

14. Tinoco, I., Adv. in Chem. Phys. 4, 113 (1962).

15. Kuhn, W., Trans. Faraday Soc. 26, 293 (1930). 